\title{
Chemotherapy- and Irradiation-Induced Bone Loss in Adults with Solid Tumors
}

\author{
Michel D. Wissing
}

Published online: 25 February 2015

(C) The Author(s) 2015. This article is published with open access at Springerlink.com

\begin{abstract}
It is estimated that bone loss occurs in $70 \%$ of all patients dying from cancer, causing a significant disease burden in cancer patients. Bone loss is caused by cancer itself and its metastases, but also by cancer therapies. Of the cancer therapy-induced bone loss, hormone therapies are best known for their bone damaging abilities. However, chemo- and radiotherapy may result in bone loss too. In this review, direct and indirect effects of various chemotherapies (such as methotrexate, imatinib, and taxanes) that cause bone loss are discussed. Furthermore, we discuss bone loss caused by radiotherapy and radionuclides, of which the latter may be reduced with the introduction of the alpha-emitter Radium-223. Finally, agents preventing chemotherapy- or radiotherapy-induced bone loss, in particular denosumab and bisphosphonates, are being reviewed for their efficacy in preventing chemotherapy- and irradiationinduced bone loss in cancer patients.
\end{abstract}

Keywords Chemotherapy-induced bone loss .

Radiotherapy-induced bone loss $\cdot$ Solid tumors .

Antiresorptive agents $\cdot$ Radionuclides

\section{Introduction}

Cancer is one of the most prevalent and deadliest diseases in the world, with an estimated 1.7 million new cases and 586,

This article is part of the Topical Collection on Osteoporosis and Cancer

M. D. Wissing $(\bowtie)$

Department of Medical Oncology, Leiden University Medical Center, Albinusdreef 2, Leiden 2333 ZA, The Netherlands

e-mail: m.d.wissing@lumc.nl
000 deaths in the USA in 2014 [1]. In cancer patients, bone loss occurs frequently: it is estimated that bones are affected of over $70 \%$ of all patients dying from cancer, often resulting in significant morbidity and mortality [2]. Bone disease primarily occurs due to bone metastases: lung carcinomas, causing most cancer deaths in both men and women [1], as well as prostate and breast cancer, the most prevalent cancers in men and women, respectively [3], frequently metastasize to the bones; other solid tumors metastasize to bones as well [2]. Furthermore, bone may be damaged in cancer patients by other causes, such as cancer therapy. For example, in a case-control study, breast cancer patients without bone metastases had a significant increase in vertebral fractures (odds ratio (OR) of 4.7) as compared to controls from a general population [4].

It is well known that hormonal suppression by hormone ablation therapy, frequently used in patients with among others prostate and breast cancer, results in osteoporosis and bone fractures due to a decrease in bone mineral density [5]. In prostate cancer patients who received long-term androgendeprivation therapy, osteoporosis rates increased from $35.4 \%$ in hormone-naive patients to $80.6 \%$ of patients treated with androgen-deprivation therapy for ten or more years [6]. In a study with 50,613 prostate cancer patients who did and did not receive androgen-deprivation therapy, androgendeprivation therapy increased the risk of fractures from 12.6 to $19.4 \%$ [7]. Similarly, hormonal therapy in breast cancer patients, particularly treatment with aromatase inhibitors such as letrozole and exemestane, has been found to increase the risk for osteoporosis and (pathological) fractures $[8,9]$. Considering the role of hormones in bone physiology, aforementioned increased occurrences of bone loss and skeletal-related events after hormonal-ablation therapy is not surprising.

Treatment of cancer patients with other therapies, such as radio- and chemotherapy, may result in significant bone loss as well. These therapies may directly target the bones or may 
provoke bone loss by indirect systemic effects. Furthermore, agents currently administered to cancer patients aiming to reducing bone-related adverse events may actually result in osteonecrosis. In this review, the prevalence and (potential) mechanisms of bone loss after administration of chemotherapy and irradiation will be discussed. Furthermore, novel modalities that may reduce chemotherapy- or irradiation-induced bone loss will be reviewed.

\section{Chemotherapy and Bone Loss}

Chemotherapy may lead to bone damage via indirect systemic effects, of which the most studied effect is the loss of ovarian function in women. In one study, adjuvant chemotherapy with cyclophosphamide, methotrexate, and fluorouracil in premenopausal women with breast cancer resulted in chemotherapyinduced amenorrhea in $68 \%$ (95\% CI 66-70\%) of these patients [10]. This ovarian failure resulted in rapid bone loss: within 2 years, this combination of chemotherapy resulted in bone loss of $9.5 \%$ in the lumbar spine and $4.6 \%$ in the femoral neck [11]. Other combinations of adjuvant chemotherapy induce amenorrhea in premenopausal breast cancer patients as well $[12,13 \bullet]$.

However, chemotherapy may also have a direct impact on bone (re)modeling. As summarized by Hadji et al., studies evaluating adjuvant chemotherapy in premenopausal breast cancer patients consistently reported a decrease in bone mineral density during the first year after initiation of therapy [13•]. For example, one study with premenopausal breast cancer patients reported that bone mineral density in the spine and hips of women during 6 months' adjuvant systemic chemotherapy was decreased by $1.01-1.05 \mathrm{~g} / \mathrm{m}^{2}$, independently of changes to ovarian function or amenorrhea [14].

Imatinib, used for the treatment of gastrointestinal stromal tumors and leukemia, directly targets various receptors that play a role in the bone microenvironment, such as the platelet-derived growth factor (PDGF) receptor and the macrophage colony stimulating factor (c-Fms) receptor $[15,16]$. In manipulating these receptors, bone formation was found to be increased by increasing osteoblast activity at metaphyseal osteochondral junctions and by eliminating osteoclasts from these junctions, leading to decreased bone resorption at the growth plate [17]. On the other hand, imatinib increased osteoclast activity at distal trabecular bone, resulting in increased bone resorption [17].

Many chemotherapies such as taxanes cause myelosuppression [18, 19]. Recently, Quach et al. reported that myelosuppression resulted in bone loss in mice by increased bone resorption, which was associated with increased expression of monocyte chemoattractant protein 1 (MCP1) and other inflammatory cytokines [20•]. MCP1 was also found to be increasingly expressed in cancer patients who had recently received chemotherapy and had bone loss. Inhibition of osteoclast activity by zoledronic acid prevented this MCP1-associated bone loss [20 ].

Methotrexate, used for the treatment of, among others, breast cancer, lung cancer, head and neck cancer, choriocarcinoma, and osteosarcoma, directly targets bone tissue too. In an in vivo experiment, the anti-metabolite increased apoptosis of osteocytes by a 4.3 -fold, while increasing the number of osteoclasts by a 1.8 -fold, associated with increased expression of the inflammatory cytokines IL- 6 and IL-11 [21]. These changes resulted in a $35 \%$ loss of trabecular bone. In a different study, it was found that methotrexate-induced changes to bone (re)modeling may occur due to decreased activation of the $\mathrm{Wnt} / \beta$-catenin signaling pathway [22].

Finally, corticosteroids such as prednisone play an important role in the treatment of patients with, among others, prostate cancer and multiple myeloma. Although the antitumor activity of this glucocorticoid as a single agent is being debated in most tumors, it is regularly administered concomitantly with other antitumor agents, such as taxanes and abiraterone. In multiple myeloma, the antitumor efficacy of corticosteroids by themselves is evident due to its ability to inhibit IL-6 production and to induce apoptosis in plasma cells [23]. However, prednisone directly affects bone tissue: osteoblast activity is reduced by decreased osteoblast cell replication and differentiation, while apoptosis is increased; osteoclastogenesis is increased by increased expression of RANK-ligand and decreased expression of osteoprotegerin, a decoy receptor of RANK-ligand [24]. Furthermore, urinary excretion of calcium is increased, while intestinal absorption of calcium is inhibited, resulting in further bone damage [23].

\section{Radiotherapy and Bone Loss}

On the contrary to chemotherapy, which causes bone loss both by direct and indirect effects, radiation primarily causes direct damage to bone tissue. In pediatrics, most damage from radiotherapy occurs to the growth plate, causing growth disturbances, while in adults, osteopenia and incomplete healing of bone damage results in skeletal-related events such as insufficiency fractures [25]. The exact pathogenesis of radiotherapyinduced bone loss has not been unraveled completely yet. Consensus exists that radiation decreases the number of active osteoblasts by arresting these in the cell cycle, altering their ability to differentiate, and sensitizing the cells to apoptosis $[26,27,28 \cdot]$. As summarized by Chandra et al., the role of radiation therapy in the activation of osteoclasts is still being debated, with some studies reporting a decrease in osteoclast activity while others report an increase in number and activity of osteoclasts [28•]. Nevertheless, the hypothesis that radiotherapy may induce osteoclast activity is strengthened by a clinical study that found that the addition of antiresorptive 
agents such as zoledronic acid prevented or delayed skeletalrelated events in patients with bone metastases, and by in vivo studies that indicated a decrease in radiotherapy-induced bone loss when mice were treated with bisphosphonates [29-31]. A recently published study suggests that PTH1-34 may prevent radiotherapy-induced bone loss by preventing apoptosis of osteoblasts and osteocytes [28•].

\section{Radionuclides and Bone Loss}

Radionuclides, such as Rhenium-186, Strontium-89, and Samarium-153, localize to areas with increased bone turnover, thereby selectively targeting osteoblastic bone metastases [32, 33]. For this reason, radionuclides have been used to treat bone metastases in a variety of cancers, such as prostate and breast cancer. However, most radionuclides are beta-emitters and/or even gamma-emitters, which are moderately to highly penetrating, thereby damaging surrounding tissue as well. Indeed, it has been shown that these radionuclides result in damage of healthy bone marrow, as noted by the increase in thrombocytopenia and leucopenia [33]. Therefore, radionuclides most likely damage healthy bone tissue as well, although this has not been proven. Such damage could be reduced by making use of alpha-emitting particles, which are highly energetic but do not have a high penetrative capacity. Radium-223 chloride is such a particle. It has received approval by the United States Food and Drug Administration (US FDA) for the systemic treatment of patients with castrate-resistant prostate cancer with bone metastases in 2013. As described previously, Radium-223 emits four alpha-particles and two beta-particles during its decay, until it stabilizes as Lead-207, thereby selectively targeting cells in its direct surroundings [34•]. Radium-223 increased overall survival in mCRPC patients while bone marrow toxicity was relatively low as compared to other radionuclides [35]. Nevertheless, these results need to be confirmed in studies assessing long-term efficacy and toxicity of radium-223 treatment. Currently, clinical trials are being performed to study the antitumor efficacy in patients with cancers metastasized to bones other than prostate cancer, and in patients with primary bone cancer.

\section{Agents Used for the Prevention of Bone Loss}

It is generally thought that the key to cancer-induced bone loss is an increase in osteoclast activity, resulting in decreased bone mass. Over the past two decades, bisphosphonates and the RANK ligand inhibitor denosumab have become available to prevent both cancer-induced bone loss and cancer therapy-induced bone loss. Bisphosphonates reduce osteoclast activity, thereby increasing bone mass, resulting in increased strength of the bone and a reduction in pathological fractures $[36,37]$. Various bisphosphonates have been approved for bone-related diseases, including ibradronic acid, pamidronic acid, risedronate, and zoledronic acid for the reduction of skeletal-related events in cancer patients and for patients with multiple myeloma. Of these, zoledronic acid is most commonly used, as various studies in patients with cancer-related bone disease indicated superiority of zoledronic acid over other bisphosphonates [38-40]. Treatment with bisphosphonates decreases pain secondary to bone metastases, pathological fractures, and other skeletal-related events, thereby improving quality of life [41-43].

Denosumab is a subcutaneously administered, monoclonal antibody approved by the US FDA for the treatment of unresectable giant cell tumor of bone in adults and skeletally mature adolescents, for cancer patients at high risk for fracture for example due to androgen-deprivation therapy or adjuvant aromatase inhibitor therapy, and for the prevention of skeletalrelated events in patients with bone metastases from solid tumors [44]. In various phase III studies with patients with bone metastases from solid tumors, denosumab was more effective in delaying or preventing skeletal-related events and pain progression than bisphosphonates [45-49]. In prostate cancer patients, denosumab also reduced the risk of symptomatic skeletal events, a biomarker considered more accurate for assessing clinical benefit in patients [50 $]$. Furthermore, in patients with metastatic lung cancer, overall survival was improved when patients were treated with denosumab as compared to zoledronic acid [51]. However, due to its higher cost, the cost-effectiveness of denosumab as compared to bisphosphonates remains unclear, and many physicians continue to treat cancer patients with bone disease with bisphosphonates [52].

Although bisphosphonates and denosumab aim to reduce bone disease, these agents may also cause bone damage, including hypocalcaemia, atypical femur fractures, and osteonecrosis of the jaw [37, 53]. Osteonecrosis of the jaw occurs in an estimated $7 \%$ (range $0-27.5 \%$ ) of all patients treated with bisphosphonates; its mean incidence was $1.7 \%$ in recent studies in which patients were treated with denosumab but did not differ significantly from the incidence of osteonecrosis of the jaw after treatment with bisphosphonates. Although this painful and potentially debilitating adverse event may initially be treated with antibiotics, the damage is often irreversible for which surgical management is needed. It is hypothesized that osteonecrosis of the jaw after therapy with antiresorptive agents is caused by oversuppression of osteoclast activity and/or by compromising of angiogenesis, thereby resulting in bone ischemia and sclerosis [54]. Other factors may contribute to osteonecrosis of the jaw, such as infections, poor oral hygiene, surgery to the jaw bones, diabetes mellitus, smoking, dental extraction, and concurrent medications like 
glucocorticoids or antiangiogenic medication (among others bevacizumab, sunitinib, sorafenib, mTOR inhibitors) [54, $55 \bullet \cdot]$. Indeed, recent studies have indicated that the incidence of osteonecrosis of the jaw during therapy with bisphosphonates or denosumab can be decreased by improving oral hygiene, by eliminating or stabilizing oral disease before initiating treatment, and by temporarily discontinuing treatment after extensive oral surgery $[53,55 \bullet \bullet]$.

Other agents have been or are currently being investigated for their use in the prevention of bone loss, with limited success. For example, studies are ongoing to investigate the use of gonadotropin-releasing hormone agonists such as triptorelin for the prevention of chemotherapy-induced ovarian failure. However, a prospective randomized clinical trial in patients with lymphoma did not find a statistically decreased risk of ovarian failure [56]. A meta-analysis of studies performed in breast cancer patients reported a significant decrease in premature ovarian failure after treatment with a gonadotropin-releasing hormone agonist (RR $0.40,95 \% \mathrm{CI}$ $0.21-0.75)$, but no effect on resumed menses [57]. A recent study confirms this decrease in premature ovarian failure in breast cancer patients treated with adjuvant chemotherapy [58]. However, long-term studies need to be performed to assess whether such therapy results in a decrease in chemotherapy-induced bone disease.

\section{Conclusions}

Bone disease causes high rates of morbidity and mortality in cancer patients. It can be caused both by the tumor itself and by cancer therapy. Both hormonal therapy, chemotherapy, and radiotherapy may induce bone loss. While radiotherapyinduced bone loss is primarily caused by direct bone damage, chemotherapy-induced bone damage may be the result of direct bone targeting or by indirect systemic effects, such as decreased ovarian function. Multiple agents, such as bisphosphonates and denosumab, have become available to reduce bone damage after antitumor therapy. However, these agents may induce severe bone damage too, particularly osteonecrosis of the jaw. Further research is needed to decrease the disease burden from therapy-induced bone loss in cancer patients.

Acknowledgments The author wishes to thank Prof. Dr. Gelderblom and Eugene Kim for their input in this review.

\section{Compliance with Ethics Guidelines}

Conflict of Interest MD Wissing declares no conflicts of interest.

Human and Animal Rights and Informed Consent This article does not contain any studies with human or animal subjects performed by any of the authors.
Open Access This article is distributed under the terms of the Creative Commons Attribution License which permits any use, distribution, and reproduction in any medium, provided the original author(s) and the source are credited.

\section{References}

Papers of particular interest, published recently, have been highlighted as:

- Of importance

•• Of major importance

1. Siegel R, Ma J, Zou Z, et al. Cancer statistics, 2014. CA Cancer J Clin. 2014;64:9-29.

2. Coleman RE. Clinical features of metastatic bone disease and risk of skeletal morbidity. Clin Cancer Res. 2006;12:6243s-9.

3. DeSantis CE, Lin CC, Mariotto AB, et al. Cancer treatment and survivorship statistics, 2014. CA Cancer J Clin. 2014;64: 252-71.

4. Kanis JA, McCloskey EV, Powles T, et al. A high incidence of vertebral fracture in women with breast cancer. Br J Cancer. 1999;79:1179-81.

5. Rizzoli R, Body JJ, Brandi ML, et al. Cancer-associated bone disease. Osteoporos Int. 2013;24:2929-53.

6. Morote J, Morin JP, Orsola A, et al. Prevalence of osteoporosis during long-term androgen deprivation therapy in patients with prostate cancer. Urology. 2007;69:500-4.

7. Shahinian VB, Kuo YF, Freeman JL, et al. Risk of fracture after androgen deprivation for prostate cancer. N Engl J Med. 2005;352: 154-64.

8. Pagani O, Regan MM, Walley BA, et al. Adjuvant exemestane with ovarian suppression in premenopausal breast cancer. N Engl J Med. 2014;371:107-18.

9. Rabaglio M, Sun Z, Price KN, et al. Bone fractures among postmenopausal patients with endocrine-responsive early breast cancer treated with 5 years of letrozole or tamoxifen in the BIG 1-98 trial. Ann Oncol. 2009;20:1489-98.

10. Bines J, Oleske DM, Cobleigh MA. Ovarian function in premenopausal women treated with adjuvant chemotherapy for breast cancer. J Clin Oncol. 1996;14:1718-29.

11. Saarto T, Blomqvist C, Valimaki M, et al. Chemical castration induced by adjuvant cyclophosphamide, methotrexate, and fluorouracil chemotherapy causes rapid bone loss that is reduced by clodronate: a randomized study in premenopausal breast cancer patients. J Clin Oncol. 1997;15:1341-7.

12. Walshe JM, Denduluri N, Swain SM. Amenorrhea in premenopausal women after adjuvant chemotherapy for breast cancer. J Clin Oncol. 2006;24:5769-79.

13. Hadji P, Gnant M, Body JJ, et al. Cancer treatment-induced bone loss in premenopausal women: a need for therapeutic intervention? Cancer Treat Rev. 2012;38:798-806. Tables 1 and 2 of this review contain a summary of clinical trials reporting bone loss in premenopausal patients with breast cancer.

14. Cameron DA, Douglas S, Brown JE, et al. Bone mineral density loss during adjuvant chemotherapy in pre-menopausal women with early breast cancer: is it dependent on oestrogen deficiency? Breast Cancer Res Treat. 2010;123:805-14.

15. Dewar AL, Cambareri AC, Zannettino AC, et al. Macrophage colony-stimulating factor receptor c-fms is a novel target of imatinib. Blood. 2005;105:3127-32.

16. Kubo T, Piperdi S, Rosenblum J, et al. Platelet-derived growth factor receptor as a prognostic marker and a therapeutic target for 
imatinib mesylate therapy in osteosarcoma. Cancer. 2008;112: 2119-29.

17. Nurmio M, Joki H, Kallio J, et al. Receptor tyrosine kinase inhibition causes simultaneous bone loss and excess bone formation within growing bone in rats. Toxicol Appl Pharmacol. 2011;254:267-79.

18. Tannock IF, de Wit R, Berry WR, et al. Docetaxel plus prednisone or mitoxantrone plus prednisone for advanced prostate cancer. $\mathrm{N}$ Engl J Med. 2004;351:1502-12.

19. de Bono JS, Oudard S, Ozguroglu M, et al. Prednisone plus cabazitaxel or mitoxantrone for metastatic castration-resistant prostate cancer progressing after docetaxel treatment: a randomised open-label trial. Lancet. 2010;376:1147-54.

20. Quach JM, Askmyr M, Jovic T, et al. Myelosuppressive therapies significantly increase pro-inflammatory cytokines and directly cause bone loss. J Bone Miner Res. 2014; doi: 10.1002/jbmr.2415. This study found that myelosuppresive chemotherapies directly cause bone loss.

21. Shandala T, Shen NY, Hopwood B, et al. The role of osteocyte apoptosis in cancer chemotherapy-induced bone loss. J Cell Physiol. 2012;227:2889-97.

22. Georgiou KR, King TJ, Scherer MA, et al. Attenuated Wnt/betacatenin signalling mediates methotrexate chemotherapy-induced bone loss and marrow adiposity in rats. Bone. 2012;50:1223-33.

23. Tipples K, Robinson A. Optimal management of cancer treatmentinduced bone loss: considerations for elderly patients. Drugs Aging. 2011;28:867-83.

24. Canalis E, Delany AM. Mechanisms of glucocorticoid action in bone. Ann N Y Acad Sci. 2002;966:73-81.

25. Williams HJ, Davies AM. The effect of X-rays on bone: a pictorial review. Eur Radiol. 2006;16:619-33.

26. Matsumura S, Jikko A, Hiranuma H, et al. Effect of X-ray irradiation on proliferation and differentiation of osteoblast. Calcif Tissue Int. 1996;59:307-8.

27. Szymczyk KH, Shapiro IM, Adams CS. Ionizing radiation sensitizes bone cells to apoptosis. Bone. 2004;34:148-56.

28. Chandra A, Lin T, Tribble MB, et al. PTH1-34 alleviates radiotherapy-induced local bone loss by improving osteoblast and osteocyte survival. Bone. 2014;67:33-40. Provides a summary of studies in the introduction regarding radiotherapy-induced changes in osteoclast number and activity. Indicates that PTH1-34 may alleviate radiotherapy-induced bone loss by improving osteoblast and osteocyte survival.

29. Manas A, Casas F, Ciria JP, et al. Randomised study of single dose ( 8 Gy vs. $6 \mathrm{~Gy}$ ) of analgesic radiotherapy plus zoledronic acid in patients with bone metastases. Clin Transl Oncol. 2008;10:281-7.

30. Willey JS, Livingston EW, Robbins ME, et al. Risedronate prevents early radiation-induced osteoporosis in mice at multiple skeletal locations. Bone. 2010;46:101-11.

31. Keenawinna L, Oest ME, Mann KA, et al. Zoledronic acid prevents loss of trabecular bone after focal irradiation in mice. Radiat Res. 2013;180:89-99.

32. Longo J, Lutz S, Johnstone C. Samarium-153-ethylene diamine tetramethylene phosphonate, a beta-emitting bone-targeted radiopharmaceutical, useful for patients with osteoblastic bone metastases. Cancer Manag Res. 2013;5:235-42.

33. Roque IF, Martinez-Zapata MJ, Scott-Brown M, et al. Radioisotopes for metastatic bone pain. Cochrane Database Syst Rev. 2011; CD003347.

34. Wissing MD, van Leeuwen FW, van der Pluijm G, et al. Radium223 chloride: extending life in prostate cancer patients by treating bone metastases. Clin Cancer Res. 2013;19:5822-7. Summary of radium-223 in prostate cancer patients, and the limitations of currently reported clinical studies with this novel therapy.

35. Parker C, Nilsson S, Heinrich D, et al. Alpha emitter radium-223 and survival in metastatic prostate cancer. N Engl J Med. 2013;369: 213-23.
36. Coleman RE, McCloskey EV. Bisphosphonates in oncology. Bone. 2011;49:71-6.

37. Campisi G, Fedele S, Fusco V, et al. Epidemiology, clinical manifestations, risk reduction and treatment strategies of jaw osteonecrosis in cancer patients exposed to antiresorptive agents. Future Oncol. 2014;10:257-75.

38. Rosen LS, Gordon D, Kaminski M, et al. Long-term efficacy and safety of zoledronic acid compared with pamidronate disodium in the treatment of skeletal complications in patients with advanced multiple myeloma or breast carcinoma: a randomized, doubleblind, multicenter, comparative trial. Cancer. 2003;98:1735-44.

39. Sanfilippo KM, Gage B, Luo S, et al. Comparative effectiveness on survival of zoledronic acid versus pamidronate in multiple myeloma. Leuk Lymphoma. 2014; 1-7.

40. Barrett-Lee P, Casbard A, Abraham J, et al. Oral ibandronic acid versus intravenous zoledronic acid in treatment of bone metastases from breast cancer: a randomised, open label, non-inferiority phase 3 trial. Lancet Oncol. 2014;15:114-22.

41. Mhaskar R, Redzepovic J, Wheatley K, et al. Bisphosphonates in multiple myeloma: a network meta-analysis. Cochrane Database Syst Rev. 2012;5, CD003188.

42. Wong R, Wiffen PJ. Bisphosphonates for the relief of pain secondary to bone metastases. Cochrane Database Syst Rev. 2002; CD002068.

43. Wong MH, Stockler MR, Pavlakis N. Bisphosphonates and other bone agents for breast cancer. Cochrane Database Syst Rev. 2012;2, CD003474.

44. FDA Approval for Denosumab [internet]. United States Food and Drug Administration, Silver Spring, MD, USA. Available from http://www.cancer.gov/cancertopics/druginfo/fda-denosumab. Accessed 15 Dec 2014

45. Henry D, Vadhan-Raj S, Hirsh V, et al. Delaying skeletal-related events in a randomized phase 3 study of denosumab versus zoledronic acid in patients with advanced cancer: an analysis of data from patients with solid tumors. Support Care Cancer. 2014;22: 679-87.

46. von Moos R, Body JJ, Egerdie B, et al. Pain and health-related quality of life in patients with advanced solid tumours and bone metastases: integrated results from three randomized, double-blind studies of denosumab and zoledronic acid. Support Care Cancer. 2013;21:3497-507.

47. Fizazi K, Carducci M, Smith M, et al. Denosumab versus zoledronic acid for treatment of bone metastases in men with castrationresistant prostate cancer: a randomised, double-blind study. Lancet. 2011;377:813-22.

48. Cleeland CS, Body JJ, Stopeck A, et al. Pain outcomes in patients with advanced breast cancer and bone metastases: results from a randomized, double-blind study of denosumab and zoledronic acid. Cancer. 2013;119:832-8.

49. Smith MR, Saad F, Coleman R, et al. Denosumab and bonemetastasis-free survival in men with castration-resistant prostate cancer: results of a phase 3 , randomised, placebo-controlled trial. Lancet. 2012;379:39-46.

50. Smith MR, Coleman RE, Klotz L, et al. Denosumab for the prevention of skeletal complications in metastatic castration-resistant prostate cancer: comparison of skeletal-related events and symptomatic skeletal events. Ann Oncol. 2014. First study to show that denosumab is superior in preventing symptomatic skeletal events as compared to bisphosphonates, a clinically more relevant endpoint than skeletal related events.

51. Scagliotti GV, Hirsh V, Siena S, et al. Overall survival improvement in patients with lung cancer and bone metastases treated with denosumab versus zoledronic acid: subgroup analysis from a randomized phase 3 study. J Thorac Oncol. 2012;7:1823-9.

52. Xie J, Namjoshi M, Wu EQ, et al. Economic evaluation of denosumab compared with zoledronic acid in hormone-refractory 
prostate cancer patients with bone metastases. J Manag Care Pharm. 2011;17:621-43.

53. Gartrell BA, Coleman RE, Fizazi K, et al. Toxicities following treatment with bisphosphonates and receptor activator of nuclear factor-kappaB ligand inhibitors in patients with advanced prostate cancer. Eur Urol. 2014;65:278-86.

54. Allen MR, Burr DB. The pathogenesis of bisphosphonate-related osteonecrosis of the jaw: so many hypotheses, so few data. J Oral Maxillofac Surg. 2009;67:61-70.

55.• Khan A, Morrison A, Hanley D, et al. Diagnosis and management of osteonecrosis of the jaw: a systematic review and international consensus. J Bone Miner Res. 2014. Important systematic review discussing osteonecrosis of the jaw after treatment with antiresorptive agents.
56. Demeestere I, Brice P, Peccatori FA, et al. Gonadotropin-releasing hormone agonist for the prevention of chemotherapy-induced ovarian failure in patients with lymphoma: 1-year follow-up of a prospective randomized trial. J Clin Oncol. 2013;31:903-9.

57. Yang B, Shi W, Yang J, et al. Concurrent treatment with gonadotropin-releasing hormone agonists for chemotherapyinduced ovarian damage in premenopausal women with breast cancer: a meta-analysis of randomized controlled trials. Breast. 2013;22:150-7.

58. Song G, Gao H, Yuan Z. Effect of leuprolide acetate on ovarian function after cyclophosphamide-doxorubicin-based chemotherapy in premenopausal patients with breast cancer: results from a phase II randomized trial. Med Oncol. 2013;30:667. 